\title{
Understanding Decision-Making Needs of Open Government Data Users
}

\author{
Svati Sundara Murthy \\ University of Cincinnati \\ svati.s.murthy@gmail.com
}

\author{
Jess Kropczynski \\ University of Cincinnati \\ kropczjn@ucmail.uc.edu
}

\author{
Shane Halse \\ University of Cincinnati \\ halsese@ucmail.uc.edu
}

\begin{abstract}
Open Government Data (OGD) portals make data publicly available to promote transparency, innovation, and value creation. Although these data sets are available and used by a broad audience, little is known about how users engage with this data and the websites where they are hosted. The City of Cincinnati hosts an award-winning Open Government Data Portal and is used as a case study in this paper to understand the decision-making needs of OGD end-users. The portal allows users to access local data sets such as crime reports, permits and licenses, market analysis, education/research data, viewing public safety, and public health, as part of a local OGD initiative. To investigate users' social, economical, political and other decision-making needs, this study is conducted in two steps 1) a think-aloud activity, and 2) a design iteration combined with heuristic evaluation. Observing the use of the portal through this user study provided insights into user expectations as well as system and information requirements illustrated in design implications for $O G D$ systems.
\end{abstract}

\section{Introduction}

Open Government Data (OGD) portals are data sets and platforms that allow citizens, organizations, and governments to engage with local data to ensure government accountability [1]. Open government data portals include business information, patent and trademark information, public trademark information, public tender databases, geographic information, legal information, social data, and transport information. Sharing this kind of data is intended to ensure transparency, innovation, and to improve service delivery to the users. OGD is often presented through an online portal platform which tracks activity on user accounts of how often data is accessed [1], however, there have been few accounts of how users feel about the presentation of this data in terms of its usability and accessibility. These factors are especially important to citizens' ability to make decisions using such data. OGD creates opportunities for innovation when government data sets are leveraged by third-party and citizen-created data applications, but hosting these massive data sets can also impress new barriers inherent to large-scale distributed data integration, collaborative data manipulation, and transparent data consumption [2]. This can be a large and costly undertaking for cities working on a limited budget. For this reason, gauging the usability of such tools can be take a backseat to basic operations.

The goal of this study is to better understand community perceptions of an OGD portal in terms of usability, potential for innovation, and overall value. To this end, we use The City of Cincinnati Open Government Data Portal [3] as a case study to understand OGD users' behaviors. This portal is of particular interest as it is recognized as one of the leading municipal data programs in the United States [4]. It is an interactive website where citizens, industry, and agencies have access to raw data about their local area. The City of Cincinnati Office of Performance \& Data Analytics gathers a considerable amount of data about the City in partnership with local municipal agencies [5]. It updates the information on City's Open Government Data Portal where users can download raw data and is combined with a visual tool called CincyInsights. CincyInsights is a companion tool to the data portal that was designed to visually enable citizens to know more about what is happening in their city and neighborhood using the data visualization software, Tableau (www.tableau.com).

The study takes an exploratory and grounded approach to answer the following research question: As users of OGD use the portal for decision-making, how does the usability of visual and navigational elements support or hinder activities?

The contribution of this study is to investigate the ways that individuals use OGD portal for decision-making. We also posit design insights for 
OGD tools that can increase data accessibility. A secondary contribution is to garner insights into ways that cities are using OGD portals which can encourage the development of creative tools to engage, serve, and improve neighborhoods and residents' quality of life through increased usability of these types of platforms.

To investigate these topics, this study utilizes a think-aloud activity to observe users use of the portal directly. Using this common usability approach we gained information to further improve government accessibility, increase opportunities for local innovation, and create social impact through design iterations that increase usability of the portal. In the think-aloud activity, participants are asked to use the system while continuously thinking out loud-that is, merely verbalizing their thoughts as they move through the user interface [6]. The feedback gathered will help to understand if the following aspects of the portal: typical uses, typical use patterns, if it allows users to navigate easily and find information quickly, if it is informative for decision-making needs, and if the overall layout is engaging. This activity will also help us to identify and examine the features and elements in the OGD portal that enhances the portal's usability.

\section{Background}

\subsection{The Value of OGD}

In 2009, the White House issued an Open Government Directive requiring federal agencies to take immediate actions towards transparency, participation, and collaboration [7]. Following suit, governments around the world have released datasets they have been collected over time using information technology and systems as part of their operations. These datasets are known as Open Government Data. One stated benefit of releasing OGD is to bring more business opportunities and benefits to the local areas that they serve. According to previous literature, OGD has four value-generating mechanisms to municipalities related to its application and utilization, including transparency, participation, efficiency, and innovation [8]. It is also studied concerning four types of social benefits: enthusiasm for technology, the pursuit of novelty, rejection of authority, and community involvement. The benefits also range from transparency to public service improvement, citizen engagement to the creation of social and economic value.

Based on papers related to user's perspective, we found that open data through online government portals can improve public engagement in policy-making through enhanced levels of fact-based content from open data [9]. The usability benefits of such open data portals also have a positive impact on public sector reforms, which are under pressure driven by extended periods of austerity [10]. Stakeholder analysis and perspectives analysis show that add value to OGD by exposing the identity, power, motivations, and worldview of key actors [11]. They provide a necessary foundation of knowledge for both researchers and practitioners who need to understand the different meanings of OGD in any particular context [11].Despite the impact and benefits of the usability, previous research has demonstrated that a great deal of emphasis is placed on the availability of datasets over other aspects of these initiatives [12]. This may de-emphasize work to enhance the navigation and usability of the system itself. Others have found that OGD portals situate the government as a data custodian rather than a service provider [13, 14]. Although studies have examined policies [13, 15], data sources[12, 16], theoretical research on data publics [17], efforts to promote OGD portals on social media [18], and the use of these tools by technology-savvy users [19], we found that there is very limited research related to the feedback on the performance of open data portals and the use of this data from user's perspective as they engage in local decision-making processes.

\subsection{Usability of Government Websites}

The acceptability of the websites depends on the key factor of usability i.e. if users use and return to the websites if they can easily find useful information. A good website satisfies the needs and requirements of its users and other potential stakeholders [20]. An obvious benefit of the OGD portal is its ability to enhance evidence-based decision-making by making data readily accessible [21]. Usability research is aimed to increase the use and adoption of technology by making its function intuitive to the user [10]. Assessment of the ease-of-use of OGD portal features by users can improve the use of government open data. Countries like the US and UK have taken all the necessary steps to improve the availability and ease of use of data, but there are still various barriers which revolve around human, organizations and technological factors to accessibility and usability of data which prevents its widespread use [9]. The major challenge in the adoption of OGD portals is that many scholars lack the support for the active engagement of stakeholders [9] and lack the appropriate tools to access desired information [19, 16]. It is also found that the creation of appropriate open data portals can promote transparency and openness through the publication of government data [22]. 
We operationalize transparency here as the act of making this information available, however, as is the case in open governments portals that make records transparent, there is no guarantee that available information will meet users' information needs or be used in decision-making. This study will investigate this type of transparency to learn more about how it contributes to open data users' local decision-making.

\section{Case Study: Cincinnati Open Government Data Portal and CincyInsights}

The Open Government Data Portal provides us the City's raw data sets in various fields such as economy, traffic reports, crime, and business [3]. It is an award-winning Open Givernment Data Portal [23] that provides real-time information about various services (such as recycling),initiatives (such as zoning or crime prevention), or local awareness (such as crime and health). CincyInsights is a companion dashboard that updates this data into more accessible visual trends (such as those seen in ??) than the raw data in the open data portal presented in database formats and .csv files.

The CincyInsights companion site was an explicit design to enhance user's ability to interact with data without data analysis skills. In addition to making data more accessible, these tools can be useful in helping city employees save time addressing open records requests. All CincyInsights pages contain fully interactive, automatically updated dashboards where each page provides relevant context and explanation, and includes definitions for the data in each visualization [4]. Users can easily interact and analyze the mapped data. Five major strategic priorities of both the websites are Safer Streets, Growing Economy, Thriving and Healthy Neighborhoods, Innovation Government, and Fiscal Sustainability among others

Although these tools have been designed with specific purposes in mind many of these tools have been developed on a limited budget [4] and without the time to garner a great deal of citizen feedback. This study investigates the use of these tools by users in order to learn more about how they can be adapted for local decision-making. Based on our analysis we attempt design iterations to enhance navigation and data wayfinders to support these practices by users.

\section{Methods}

\subsection{Think Aloud Protocol}

Usability testing was carried out on Cincinnati open government data portal We used this particular case study as the City of Cincinnati Open Government data portal was awarded by the Sunlight Foundation[23] and is rated highly amongst it's peers. This study demonstrates that although this is a highly rated open government portal, there could be issues with the usability and the way data is presented. To investigate further, this study intends to better understand how users navigation through the website along with the content they are using, therefore, we found a think-aloud methodology to be the ideal tool in our approach[6].Think Aloud activity is considered to be a "high cognitive load" interfere with verbalization [24]. It is one of the most effective ways to assess higher-level thinking processes that it could also be used to study individual differences in performing the same task [25]. Users were asked what their concerns were with the site and what they expected from the site. We targeted users to get answers for questions such as "Can users navigate to important information from the home page?" and "Is the data available on the portal adequate for the tasks users wish to complete?" Each session lasted about one hour. Participants were enrolled saturation of results was reached i.e. until we derived insights and common design recommendations from observations. Participants were audio-recorded to aid researchers in the qualitative coding of statements. Participants were recruited in two categories: Superusers (users who were aware, and generally used the portal often) and non-superusers(users who have never used the portal). The superusers were asked about tasks that they usually perform with the portal - they were asked to perform those tasks and explain what they're doing. Non- superusers were given tasks. For example, the participant was asked to imagine that they're deciding which neighborhood they'd like to move into and make their decision using the portal. In addition to an understanding of how users carry out tasks, questions like "If there was one thing you liked about the portal, what is it? Why?", "if there is one thing that you dislike about the portal, what is it? Why?" and "How was the overall experience of using the site?" was asked and discussed at the end of the session.

The investigation was conducted to focus on the various features, tools, sections and uses of the portal as users attempted to make a decision, along with how it would improve the overall usability of the system. Individuals were recruited from local community groups and registered users of the Cincinnati Open Data Portal. The target population were users aged above 18 years living in Cincinnati. The study was carried out by performing a think-aloud activity - an activity considered to be the most valuable and robust usability method that gives the research team a direct exposure to 
how users think according to the Nielsen Norman Group [10] to gather information on the portal. It allows users to navigate easily and find information quickly, to find the site informative for decision-making needs, and if the overall layout is engaging. When we conducted the usability test on a single user, we found major usability problems in the system. As we conducted the study with the second, third, fourth, and so on, it was observed that the more and more users we tested on, the less and less was learned about the system because we kept seeing the same things again and again [10]. Hence, testing five users for this type of study is ideal according to the Nielsen Norman Group [10] but we performed the study on 8 users until we found a pattern of similar codes. We started to reach out to the non-superusers from the University of Cincinnati - Faculty members and students who were using the City of Cincinnati Open Government Data portal for the first time.Though they never used open government data, we recruited participants who could use web services well in general. This activity took place for about an hour and was conducted in university labs.

Qualitative Thematic analysis (where data is manually reviewed, patterns are searched and linked together in the form of themes) were performed to examine relationships between participants' community perceptions regarding values such as usability, innovation, and transparency. The participants for the research were observed while they used the portal and were asked to explain what they are doing during the process. The participants were given detailed instructions before the activity began.They were also required to sign a consent form that states that they are 18 years and above and are willing to participate in the study. The session was held for around an hour, and a detailed report was generated at the end of each user performing each task. Questions were asked while the participants use the City of Cincinnati portal to test the usability of the website and if it provides an experience that covers 5 E's of usability as defined by Whitney Quesenbery [26] - effective, efficient, engaging, ease of use and error-tolerant. By doing this, we understand how precisely the study is completed, how fast the study has been completed, how enjoyable the interface is, how the product prevents errors and helps the user to recover mistakes and how well the system supports continuous learning through the complete lifetime of use [26]. The interview script included in this study is included in the Appendix.

Superusers, or users who actively use Cincinnati Open Data portal, were asked to work with the portal in a way that they usually engage with it - preferably one or two tasks that they typically perform and provide immediate feedback on the usability of the portal. This stage helped us analyze the drawbacks and improvement areas of the portal. Non-superusers were given a task or two since they do not typically use this tool. These users provided insightful information from a different user perspective as they are inexperienced with the open data portal.

\subsection{Heuristic Evaluation}

Based on the information gathered from the think-aloud activity, a heuristic evaluation was performed. The word 'Heuristic' means 'Rule of thumb' which means that it is a very large design guideline that can be applied to a broad range of user interface. This is a method of judging the usability flaws by comparing them with certain principle knows as usability heuristics. In this study we took the reference of the ten most fundamental principles that are given by the Nielsen Norman Group[27].The ten usability heuristics are:

- Visibility - The system should keep users informed of what is going on

- Match between the system and the real world The system should always use easy language that the target users can understand. This is extremely important for error messages.

- User control and freedom- Users should be able to UNDO and REDO, move backwards whenever necessary and always have an emergency exit as an option.

- Consistency and standards - Screen elements should be the same and consistent across the entire platform. For example, Microsoft Word, Excel and PowerPoint have the same header points.

- Error prevention - Careful design should be crafted so that users cant break the system and errors are at the minimum.

- Recognition rather than recall - The system should always be designed in such as way that there is minimum cognitive role of the users.

- Flexibility and efficiency of use- Shortcuts should be present as it allows to speed up the experience of the novice users

- Aesthetic and minimalist design- The system should limit the design to having the must have elements only. Irrelevant elements should not be included in the design 
- Help users recognise, diagnose and recover from errors - Error messages should use plane language without quotes to indicate the problem and suggest the solution

- Help and documentation - There should always be documentation to provide the user with additional information

In this method, a participant (examiner) was asked to evaluate and examine the common usability issues of the portal. The experimenter(the observer conducting the study) interpreted the user's action to infer how these actions are related to the usability issues in the design of the interface while the examiner has to analyze the user interface [27]. A severity rate was also provided on a scale of 5 for each violation noted. At the end of the heuristic evaluation, major points of concern in the design of the portal were listed and rated accordingly. This helps in the interactive design process as each usability violation is listed concerning the various usability principles that explain why each particular aspect of the interface element is a usability problem. The schema for the severity rating is noted below.

- $\mathbf{0}$ - don't agree that this is a usability problem

- 1 - cosmetic problem

- 2 - minor usability problem

- 3 - major usability problem; important to fix

- 4 - usability catastrophe; imperative to fix

\subsection{Data Analysis}

Data was collected, imported, and linked for analysis. Data was reviewed manually to ensure that the number of in-person meetings does not result in more than that number of responses. Additionally, open coding strategies as suggested by Strauss [28] were applied to the data to develop a thematic coding scheme of commonly occurring patterns in users' experience. Themes and concepts were identified, discussed, and refined iteratively among researchers [29]. Preliminary data was assigned to the data to describe the content. Patterns were searched in these codes after saturation. A detailed report was produced after naming and defining these themes. Through values such as transparency,innovation, and usability, we may be able to find a way to improve government accessibility, increase opportunities for local innovation, and create social impact.

\section{Results}

Studies were conducted on 8 participants referenced in this section as $\mathrm{P} 1, \mathrm{P} 2$, etc. Here, the superusers and the non-superusers were made to think aloud as they perform various tasks designed, keeping in mind the endpoints of this study. While the participants performed these tasks, our research team observed and noted down every action and word said by the participants. This helped us gain information with regards to what the users felt while performing the tasks as well as the strong and weak points of the portal itself. Individual's signed informed consent was gathered from these individuals before they proceeded to complete the activity

\subsection{Searchability}

The first key theme when participants began using the system is the need to find the information they were looking for. P6 commented: "I didn't know that there was a search icon, I'm seeing it now. Its small box placed in the corner that went unnoticed-I just used the blue icons". P1, P2, and P5 and P7 also made use of the big icons present on the main page for navigation. P1 and P4 also made use of the A-Z bar shown in Figure 2 instead of the search bar. P4 commented: "A-Z is good. If at all I don't know what I'm looking for, I would use the a-z dashboard to see what information this website provides. The A-Z dashboard is very helpful."

\subsection{Explanations of Key Datasets}

As most of the users were using the portal for the first time, they were unaware of what many sections tell or represent. P1 told us that related links were not present below each section to dive further for better understanding. P3 and P5 preferred a small description at the beginning of each section that would help in a better understanding of what the page is about. P5 was not very well-versed with open government data and preferred to have a description or a link that directs to a detailed explanation page of what OGD is.P5 also commented on certain sections of the portal saying: "Private lot abatement program. What does the abatement program mean?- I honestly have no idea. I don't know what all this means".

Each section of the webpage had statistical information, maps, and tables without any information at the beginning of the page. This made the users skip several pages that they did not understand. 


\subsection{Geographic Referencing in Maps}

P1, P2, P3, P4, and P5 faced major issues with the maps present in each section. P1 and P2 found that the maps present in each section were unclear and not intuitive. P3 preferred the maps to be more usable, for example, this participant preferred heat spots on the crime data map that would help in identifying the density of the crime in each area immediately. P3 mentioned:

"I zoom in the map and see a lot of individual crimes. The way it's displayed-if there was some kind of a heat map that would show you the crime hotspotsright now it's just a lot of dots and I can see where it's concentrated, but it's not giving me a high-level view of what I'm looking at". P4 found that the maps section could not be expanded in a full-screen mode and also preferred to have the google maps embedded that would make the maps more clear and easy to use. P4 commented: "Maps were not intuitive. If google maps would be embedded, it would have been good. If I zoom into the google maps, it shows me the name of the area, what parks are there, etc which does not appear here. All the dots placed on this map are very close and almost overlapping". P5 also preferred to have the option of expanding the maps into full-screen mode.

\subsection{Data Comparison Features}

One of the tasks that were given to the users was to make their decision about which neighborhood they would choose to move in by utilizing the data in the OGD portal. During this task, users preferred to have a "select multiple option" feature and a "compare" feature that would help them to filter out their preferences and needs easily. P3 and P7 preferred this compare option that would help decision-making more efficient. P3 comments were: "It's easy to compare the reported crime but it's harder to compare the types of crimeyou have to keep clicking back and forth. It's probably beyond their scope of what they are capable, but if they added some way to compare, something like a plus sign where you can select multiple communities and it would give you some sort of aggregate and comparative statistics, that would be good."

P7 stated "I wish there was just a tabular column at the corner which would allow me to compare different neighborhoods based on my needs." P5 also wanted the option of comparing two or more neighborhoods based on crime, public health, etc.

\subsection{Selection of Keywords for Basic Search}

While P4 searched for words like "hospitals" from the search bar menu, the results repeatedly displayed as an unsatisfying "No results found". After several trials, only "help centers" showed the results of hospitals that the participant was looking for.P4's comments were: "What I don't like about the website is, when I searched for hospitals in the search bar, it didn't show up because it was mentioned as health centers. That shouldn't happen. Hospitals should also be coming under health centers. Based on this I might just compute that this website is not helpful to search for hospitals. Keywords that are similar to a word should lead me to that particular search topic." The user mentioned that keywords should have been implemented that would have helped in navigation. P6 also received blank pages when the user typed in regional keywords like "Clifton" and "Hyde Park" which are local neighborhood names. P7 was also very disappointed when several searches only showed up as "no results found".

Once these Think-aloud events were completed,the participants were thanked for their cooperation and invited for optional participation in the heuristic evaluation of a design iteration.

\subsection{Heuristic Evaluation Results}

After the think-aloud activity was conducted, the user who faced maximum problems was selected to evaluate an iteration on the design. We examined the same task, screen by screen, and compared the original screenshots with the improved ones. Common violations from the feedback gained by the users were noted. Jakob Nielsen's Ten Usability Heuristics [30] was selected to examine iterations to the design. Each item was discussed and a severity rating marked for each that indicates the results of the discussion and is noted beside the criteria in Table 1.

The Difficulty understanding what the subsection is about field was marked 4 as majority of the users navigated to different pages to see that they did not understand the statistics nor the topic. A brief description would have drastically helped the users to understand better. Maps cannot be expanded into full screen field was given a severity of 3 as the majority of the users navigated to different pages to see that they did not understand the statistics nor the topic. A brief description would have drastically helped the users to understand better. "No results found" was given a severity of 4 . This was a major usability catastrophe as most of the users used the search icon to search for hospitals, supermarkets, or neighborhoods. Users were 
Table 1. Results of Heuristic Evaluation

\begin{tabular}{|c|c|c|}
\hline Violation & Heuristic & Severity \\
\hline Difficulty understanding what the subsection is about & Match between the system and the real world & 4 \\
Maps cannot be expanded into full screen & Flexibility and Efficiency of use & 3 \\
No results found" for most of the searches & Recognition rather than recall & 4 \\
Search toolbar not visible & Help and documentation & 2 \\
Unable to select and compare two neighborhoods & Flexibility and efficiency of use & 2 \\
\hline
\end{tabular}

almost frustrated as the page constantly displayed "No results found".

In addition to the above, "Search toolbar not visible" was given a severity of 2 as this was a minor usability problem. Few users directly used the big buttons. Some users used the other huge search tool present in search tool present in the center of the page."Unable to select and compare two neighborhoods" was marked 2 as many users mentioned that would prefer a table that compares two neighborhoods. This is considered minor as this feedback was more task-orientated. Had there been a different task, this would not have been suggested by the users.

\section{Discussion}

When we compared what the superusers and non-superusers have to say about the website, we found that both the groups agreed on major points such as data accuracy and easy navigation. The major errors that both the groups agreed on are that the portal had screen size and map adaptability issues. Another important aspect that we found out was that as the superusers were well-informed about both the City of Cincinnati Open Government Data Portal and CincyInsights websites, it was difficult for them to compare data from both platforms to perform their regular tasks.

Users were generally able to find the information they were looking for in the portal through trial and error. They found the information on the portal transparent and useful to make decisions. However, many of them were unaware of all of the features of the open government data portal and only made use of a limited number of features. In addition, most of the users (non-super users) were unable to understand each page of the website without the brief description of it mentioned on the page. Although they had found this data informative and accurate, few changes in terms of usability would have made their navigation flow easier.

As a result of the above user feedback, the following remain outstanding issues in overall usability and we attempt to address these through an iteration on the current design, then follow up with a brief heuristic evaluation of the design:
- Ability to use the search icon more effectively by making it more visible.

- Ability to know a brief description of each section at the beginning of the page.

- Ability to make the maps be viewed in a full-screen mode

- Ability to easily understand the densely crime populated regions through heat spots

- Ability to select two or more neighborhoods and compare them.

- Ability to find related articles instead of "no results found" while searching any information

\subsection{Design Iteration}

The following section describes changes to the design based on constraints experienced by users.

The first and most prominent constraint face by users was searchability. Users today are accustomed to using search features to quickly navigate a site. Changes were made in the search bar - a larger and more visible search bar was placed in the top-right corner of the webpage that would not go unnoticed as seen in Figure 1
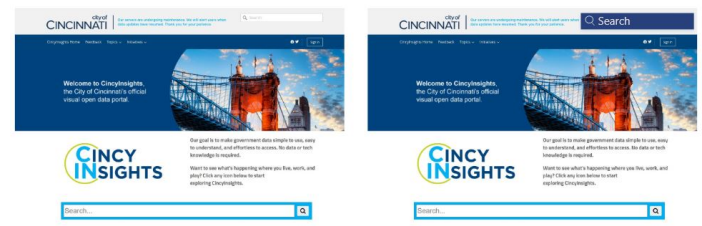

Figure 1. Larger search bar in top right corner

A second constraint is explanations of data sets wherein some users need a brief orientation to the content they are viewing, especially when specialized government terms or policies are mentioned. For this reason, descriptions of each section were moved from the bottom of the page to the top of the page as many users preferred to read about what each section is about before diving into maps and statistics as shown in Figure 2 


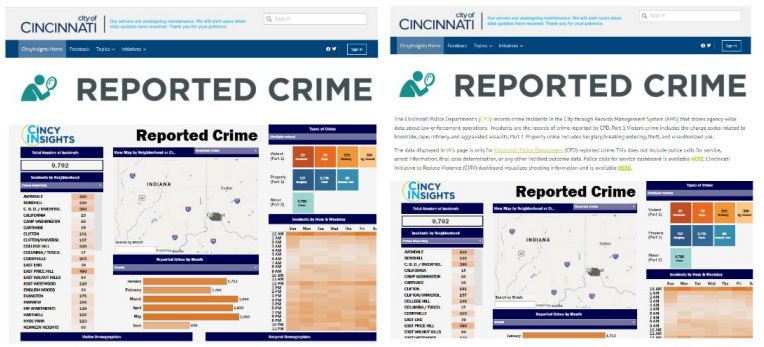

Figure 2. Each section description positioned at top

A third constraint is that users sometimes struggled with geographic referencing in maps as presented in pinpoints. Hot spots of a pattern on the map were added to allow the user to identify the denser areas in crime immediately as shown in Figure 3.
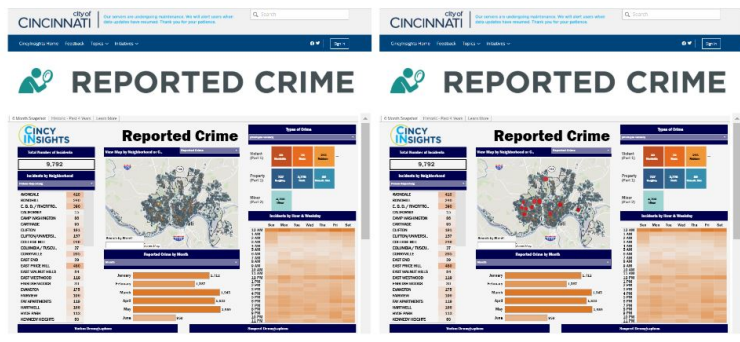

Figure 3. Map hotspots to make patterns evident

Another issue that users had with the maps was the relative size, therefore we recommend that maps have embedded google maps which can be zoomed in and adaptable to full-screen mode for a better view as shown in Figure 4

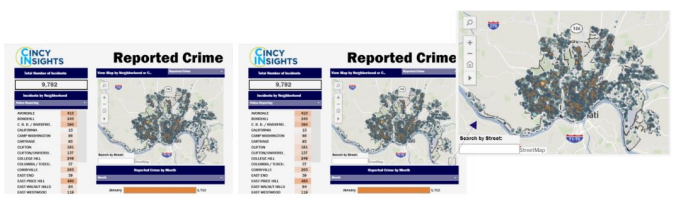

Figure 4. Embedded adaptable Google map

A fourth constraint is that users desired additional data comparison features, therefore comparison tables were added through which two or more neighborhoods can be compared based on the type of crimes as shown in Figure 5.

Finally, a fifth constraint that users faced is that users were unsatisfied with seeing that no results were found on pages based on selection of keywords for basic search. On the backend, search terms need to be added to pages such as neighborhood names and common references according to a user perspective. On

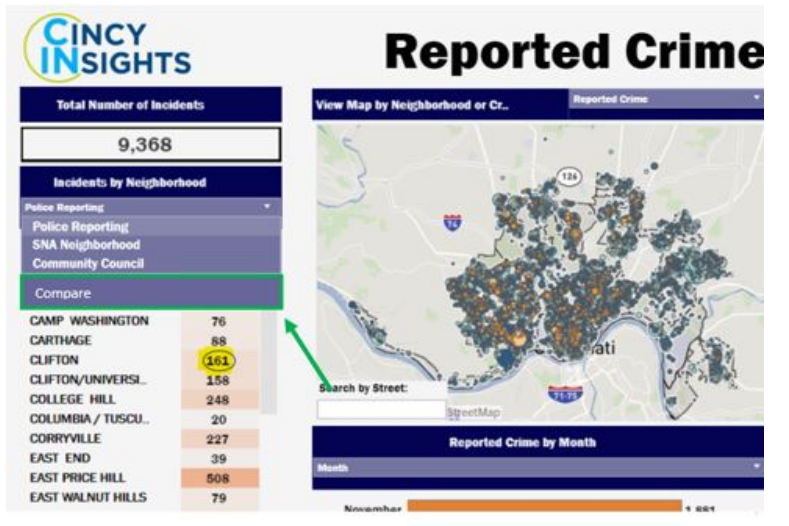

\section{(2) Reported Crime}

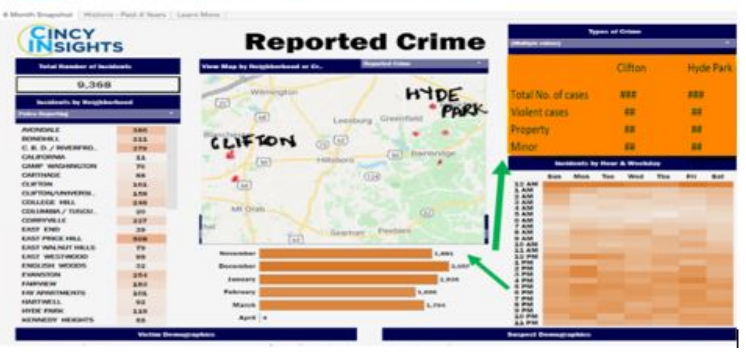

Figure 5. Comparison tables of crimes.

the front end, we recommend that related pages are displayed instead of a common and discouraging "no results found" while searching for information as shown in Figure 6.

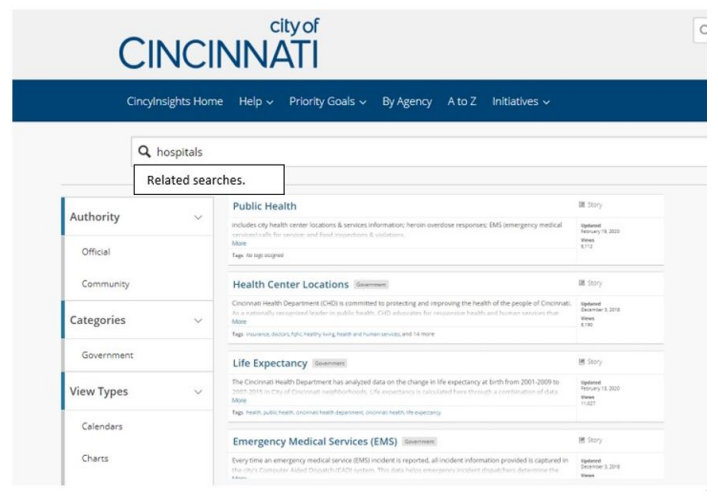

Figure 6. Related page to search

These results and design implications tell us that open government portals still come across usability flaws. The effects of open data utilization are potentially far reaching for sustainable development with a positive impact on innovation, transparency,accountability, participatory governance and economic growth [31]. These insights are valuable to both practitioners 
to develop more usable wand academics to further improve the usability of the OGD portals. This data which is open to the public should be presented in form that considers the capabilities and skills of the general public to understand, use and gain insights from it.

\subsection{Limitations and Future Work}

In this study we present these initial findings as a starting point for understanding methods to increase usability and adoption of such resources into regular user practices that may not be generalizable to other users or other OGD portals. The study is conducted on the City of Cincinnati open government data portal which is already an award-winning open government data portal. Thus, the user's experience with this portal might be different from those of other portal users.In next steps of this study we intend to use survey research to understand the broader experience of users and identify root problems faced by citizens while using the portal. Given responses to the user study presented in this paper, Likert scales, multiple-choice questions (MCQs), and open-ended questions can be better developed in order to capture common users experiences. Information gathered from this survey will allow us to observe whether the patterns observed in the Think Aloud that are generalizable to the larger population of users and will contain a variety of perspectives along with it being rich in data since the survey is conducted on a large scale.

\section{Conclusion}

In this study, we have examined the usability of an open government data portal in supporting citizens' decision-making needs while viewing local data. Our results highlight several factors that may hinder basic usability of OGD. Our results also indicate that searchability, explanations of key datasets, geographic referencing, comparisons, and selection of keywords for basic search were at the forefront of users' experience and warrants future work to understand other factors that may support wide-scale adoption of the use of these resources. Based on our findings, we believe that design iterations may be useful to investigate the usability of open government data tools. In addition, our think-aloud activity and heuristic analysis approach has addressed usability flaws that may be critical to the design considerations of OGD tools. One important contribution from utilizing this method is an understanding of how citizens navigate these tools and potential design implications for other OGD portals.

\section{References}

[1] J. Thorsby, G. N. Stowers, K. Wolslegel, and E. Tumbuan, "Understanding the content and features of open data portals in american cities," Government Information Quarterly, vol. 34, no. 1, pp. 53-61, 2017.

[2] L. Ding, T. Lebo, J. S. Erickson, D. Difranzo, G. T. Williams, X. Li, J. Michaelis, A. Graves, J. G. Zheng, Z. Shangguan, et al., "Twc logd: A portal for linked open government data ecosystems," Journal of Web Semantics, vol. 9, no. 3, pp. 325-333, 2011.

[3] Cincinnati, "Cincinnati open data portal." https:// data.cincinnati-oh.gov/, n.d. (Accessed on $01 / 28 / 2020$ )

[4] Z. Quaintance, "The cincyinsights data program continues to raise the bar." https://bit.ly/ 3gVC5h8, February 2019. (Accessed on 07/10/2020).

[5] Z. Quaintance et al., "What's new in civic tech: States deploy chatbots for crisis," 2020.

[6] J. Nielsen, "Thinking aloud: The \#1 usability tool." https://www.nngroup.com/articles/ thinking-aloud-the-1-usability-tool/ January 2012. (Accessed on 05/10/2020).

[7] T. W. House, "Open government: A progress report to the america people," (Accessed on 09/19/2019).

[8] T. Jetzek, M. Avital, and N. Bjorn-Andersen, "Data-driven innovation through open government data," Journal of theoretical and applied electronic commerce research, vol. 9, no. 2, pp. 100-120, 2014.

[9] V. Weerakkody, Z. Irani, K. Kapoor, U. Sivarajah, and Y. K. Dwivedi, "Open data and its usability: an empirical view from the citizen's perspective," Information Systems Frontiers, vol. 19, no. 2, pp. 285-300, 2017.

[10] J. Nielsen, "Nielsen norman group: Ux training, consulting, \& research." https: //www.nngroup. $\mathrm{com} /$. (Accessed on 07/15/2020).

[11] F. Gonzalez-Zapata and R. Heeks, "The multiple meanings of open government data: Understanding different stakeholders and their perspectives," Government Information Quarterly, vol. 32, no. 4, pp. 441-452, 2015.

[12] J. Corbett, M. Templier, and H. Takeda, "The making of a'top'open data city: A case study of edmonton's open data initiative," in Proceedings of the 51st Hawaii International Conference on System Sciences, 2018.

[13] P. Johnson, "Disintermediating government: the role of open data and smart infrastructure," in Proceedings of the 52nd Hawaii International Conference on System Sciences, 2019.

[14] O. Marjanovic and D. Cecez-Kecmanovic, "Understanding datafication effects of open government information systems-a contemporary systems thinking approach,” 2017.

[15] D. Rudmark, "Open data standards: Vertical industry standards to unlock digital ecosystems," in 53rd Hawaii International Conference on System Sciences, 2020.

[16] E. Folmer, W. Beek, L. Rietveld, S. Ronzhin, R. Geerling, and D. den Haan, "Enhancing the usefulness of open governmental data with linked data viewing techniques," in Proceedings of the 52nd Hawaii International Conference on System Sciences, 2019. 
[17] A. Washington, "Who do you think we are? the data publics in digital government policy," in Proceedings of the 52nd Hawaii International Conference on System Sciences, 2019.

[18] C. Born, C. Meschede, T. Siebenlist, and A. Mainka, "Pushing open government through social media," in Proceedings of the 52nd Hawaii International Conference on System Sciences, 2019.

[19] M. Young and A. Yan, "Civic hackers' user experiences and expectations of seattle's open municipal data program," in Proceedings of the 50th Hawaii International Conference on System Sciences, 2017.

[20] J. Nielsen, "Enhancing the explanatory power of usability heuristics," in Proceedings of the SIGCHI conference on Human Factors in Computing Systems, pp. $152-158,1994$.

[21] A. Ho, "Big data and evidence-driven decision-making: Analyzing the practices of large and mid-sized us cities," in Proceedings of the 50th Hawaii International Conference on System Sciences, 2017.

[22] R. Máchová, M. Hub, and M. Lněnička, "Usability evaluation of open data portals: Evaluating data discoverability, accessibility, and reusability from a stakeholders' perspective," Aslib Journal of Information Management, vol. 70, 052018.

[23] E. Miller and E. Miller, "Sunlight foundation."

[24] K. A. Ericsson and H. A. Simon, "Verbal reports as data.," Psychological review, vol. 87, no. 3, p. 215, 1980.

[25] E. Charters, "The use of think-aloud methods in qualitative research an introduction to think-aloud methods," Brock Education: A Journal of Educational Research and Practice, vol. 12, no. 2, 2003.

[26] W. Quesenbery, "Balancing the 5es of usability," Cutter IT Journal, vol. 17, no. 2, pp. 4-11, 2004.

[27] J. Nielsen, "How to conduct a heuristic evaluation," Nielsen Norman Group, vol. 1, pp. 1-8, 1995.

[28] A. L. Strauss, Qualitative analysis for social scientists. Cambridge university press, 1987.

[29] A. Lacey and D. Luff, Qualitative data analysis. Trent focus Sheffield, 2001.

[30] J. Nielsen, "Enhancing the explanatory power of usability heuristics," in Proceedings of the SIGCHI conference on Human Factors in Computing Systems, pp. 152-158, 1994.

[31] "Open government data and services."

\section{A. Think Aloud Protocol}

\section{A.1. Introduction to study}

Hi, [participant]. My name is [Researcher's name], and I'm a research assistant at [University name]. I will be asking you questions while you use the City of Cincinnati Open Data Portal to test the usability of the website. The session should take about an hour.

As you use the site, I'm going to ask you as much as possible to try to think out loud: to say what you're looking at, what you're trying to do, and what you're thinking.We're doing this to only assess the quality of the website and generate a detailed report about each user performing each task and therefore determine how usable the website is.

With your permission, we're going to record what happens on the screen and our conversation. The recording will only be used to help us figure out how a user navigates and performs the tasks given to them on the site, and it won't be seen by anyone except our team who are working on the report.If you would, I'm going to ask you to sign a simple permission form for us. It just says that you agree to be a part of our research study and we have your permission to record you, and that the recording will only be seen by the research team. We appreciate your participation in this project and hope you find this discussion interesting.

Do you have any questions about the study?

\section{A.2. Orientation to Task}

FOR SUPERSUERS: Let's come up with 1-2 tasks that you normally do with this portal. Can you tell me what they are and then I'll ask you to begin.

FOR NONSUPERUSERS: Are there any tasks that you typically do within this system that I can watch you perform? If not, I'd like to assign you a task. Imagine you're trying to decide which neighborhood you'd like to move into, please use the system to make your decision.

Please tell me about your thoughts/decisions as you proceed.

\section{A.3. Carrying out task}

Participant is asked to proceed with task outlined above and think aloud while doing so. The following probes may be used as they proceed:

- What are you thinking now?

- I noticed that you are navigating in an interesting way, why are you doing that?

\section{A.4. Closing questions}

The following questions are asked when participant has completed the task.

- Let's take a moment to reflect on your overall experience. If there was one thing that I should change for the next version and nothing else, what would you suggest that I work on?

- Open Government Data is believed to improve government accountability, increase opportunities for local innovation, and have social impact - do you think that is true of this website?

- Is there anything else you'd like to share? 